\title{
Spotlight on idarucizumab and its potential for the reversal of anticoagulant effects of dabigatran
}

\author{
This article was published in the following Dove Press journal: \\ Drug Design, Development and Therapy \\ 18 May 2016 \\ Number of times this article has been viewed
}

\section{Pierre Sié}

Hematology Laboratory, Academic Hospital of Toulouse, Hospital

Rangueil, Toulouse, France
Correspondence: Pierre Sié Hématologie Laboratoire, Centre Hospitalier Universitaire de Toulouse, Hôpital de Rangueil, Avenue du Professeur Jean Pouhlès, TSA 50032, Toulouse 31059, CEDEX 9, France

$\mathrm{Tel}+33561322289$

$\mathrm{Fax}+33561322233$

Email sie.p@chu-toulouse.fr
Abstract: Idarucizumab is the first targeted antidote of dabigatran, a direct oral anticoagulant used for prevention and treatment of venous thromboembolism and prevention of stroke in atrial fibrillation. Idarucizumab is a humanized fragment of a monoclonal antibody, which binds dabigatran reversibly with high affinity and, when administered intravenously, immediately neutralizes its anticoagulant effect. It is rapidly cleared by the kidney with captured dabigatran. In Phase I and II trials, no significant adverse events have been reported. Specifically, idarucizumab has no anticoagulant or procoagulant effect by itself. Idarucizumab is currently being evaluated in an ongoing Phase III trial, in patients treated with dabigatran presenting with severe active bleeding or requiring emergency surgery or an invasive procedure and are at high risk of bleeding. The results of the interim analysis confirm the ability of idarucizumab to neutralize dabigatran instantaneously, without rebound effect, except in rare patients with very high baseline levels of anticoagulant. Although not definitely proving clinical efficacy, due to the noncontrolled design of the trial and the heterogeneity of patient conditions, these promising results on an intermediate criterion with strong rationale have led to the approval of idarucizumab for these indications. However, several questions are unresolved. First, activity measurement of dabigatran in blood, useless in current practice, could be useful to guide the treatment and avoid over- or underutilization of the antidote; but so far, it has not been largely available in real time. Second, the translation of anticoagulant neutralization to an effect on mortality and better outcome is highly dependent on the global management of these patients, especially rapid diagnosis, supportive care, and easy access to antidote administration. Although idarucizumab represents a remarkable achievement in drug design and development, whether it will be an important step toward improved safety of patients treated with dabigatran in the real world will have to be demonstrated in the postmarketing phase.

Keywords: dabigatran, idarucizumab, bleeding, surgery

\section{Introduction}

Direct oral anticoagulants (DOACs), targeting activated coagulation proteases, have been recently introduced in therapeutics for stroke prevention in patients with nonvalvular atrial fibrillation, and prevention and treatment of venous thromboembolism; they are currently in development for various other indications. ${ }^{1,2}$ DOACs are an alternative to vitamin $\mathrm{K}$ antagonists (VKAs) for mid- or long-term antithrombotic treatments. The main limitation of VKAs is the fear of bleeding. ${ }^{3,4}$ The rate of major bleeding among long-term users of VKAs is $1.5 \%-5.2 \%$ per year, with a mortality rate from bleeding $>10 \%$. In the recent past, $>40,000$ patients included in the comparative arms of randomized controlled trials were administered VKAs up to the conventional target of international normalized ratio (INR) between two and three, for at least 3 months. The rate of major bleeding, fatal bleeding, and intracranial hemorrhages in these patients 
were $4.64 \%, 0.52 \%$, and $1.05 \%$, respectively. ${ }^{5}$ In real life, it can be speculated that this risk is superior. VKAs have several other limitations, including the need for laboratory monitoring, and in many patients, instability of anticoagulation, dietary and drug interactions, a slow onset of action, and a narrow therapeutic window. DOACs that directly inhibit coagulation factor Xa (xabans) or thrombin (dabigatran) have been developed to overcome these limitations. However, VKAs will probably be present on the market still for long time, because: 1) they have been approved for use in a larger set of thrombotic conditions compared to DOACs; 2) they can be prescribed in patients with severe renal insufficiency; and 3) dose adjustment using INR is possible when strong drug interference is anticipated.

DOACs have been developed without specific antidote on the basis of their relatively short half-life (hours) and on the assumption of improved safety compared to VKAs. Although DOACs produced a modest reduction of major bleeding in randomized trials, ${ }^{1,6}$ which seems to be reproduced in "real life", ${ }^{7-9}$ a rapid reversal strategy of the their anticoagulant effect is desirable. ${ }^{10}$ Idarucizumab is the first marketed DOAC antidote targeting dabigatran. This review provides short insights on the target drug dabigatran and the mechanism of its action, current development, potential indications, and clinical utility of idarucizumab.

\section{The target drug dabigatran}

Dabigatran is the active metabolite of dabigatran etexilate (Pradaxa ${ }^{\circledR}$; Boehringer-Ingelheim, Ingelheim, Germany). ${ }^{11}$ It is a small (471 Da) nonpeptidic inhibitor of thrombin (factor IIa), the key serine protease product of the common coagulation pathway of blood coagulation. Dabigatran and its glucuronide metabolites bind thrombin reversibly with an affinity close to $700 \mathrm{pM}$, via a salt bridge that anchors the benzamidine moiety of dabigatran to a hydrophobic cavity in the active site of thrombin. ${ }^{11}$ Dabigatran blocks thrombin's catalytic activity, but not the interaction of thrombin exosite with macromolecular substrates, including protease-activated receptors.

After oral administration, dabigatran etexilate is rapidly hydrolyzed by nonspecific, ubiquitous esterases to the active metabolite. ${ }^{12}$ Dabigatran etexilate, but not dabigatran, is a substrate for the efflux transporter P-glycoprotein. In healthy volunteers, peak plasma concentrations $\left(C_{\max }\right)$ of dabigatran and the anticoagulant effects are achieved within 1.5-3 hours after oral dosing. Dabigatran plasma concentrations decline in a biphasic manner characterized by a rapid distribution phase, followed by a prolonged terminal elimination phase, resulting in a mean plasma terminal half-life of 12-14 hours, independent of dose. The apparent volume of distribution is about $1 \mathrm{~L} / \mathrm{kg}$. Dabigatran and its metabolites are eliminated mainly by the kidney $(>80 \%)$. Increased trough concentrations are observed in patients with moderate renal insufficiency (creatinine clearance: $<50 \mathrm{~mL} / \mathrm{min}$ ) and dabigatran is contraindicated in severe renal impairment (creatinine clearance: $<30 \mathrm{~mL} / \mathrm{min}$ ). The pharmacodynamic profile is parallel to the pharmacokinetics, ${ }^{13}$ with a prolongation of the global coagulation tests (prothrombin time or activated partial thromboplastin time [aPTT]) and thrombin time (TT). ${ }^{13,14}$

Dabigatran has been evaluated at fixed doses (110 or $150 \mathrm{mg}$ bid) without laboratory monitoring in large randomized clinical trials for the prevention of venous thromboembolism after total hip or knee replacement (RE-NOVATE, ${ }^{15}$ RE-MODEL ${ }^{16}$ RE-MOBILIZE),${ }^{17}$ treatment of acute venous thromboembolism (RE-COVER ${ }^{18}$ ), and prevention of stroke and cardioembolism in nonvalvular atrial fibrillation $\left(\mathrm{RE}-\mathrm{LY}^{19}\right)$ before approval in these indications.

\section{Mechanism of action of idarucizumab}

Idarucizumab is a humanized monoclonal antibody Fab fragment designed for the reversal of dabigatran. ${ }^{20,21}$ A similar approach has been successfully used for the neutralization of other drugs (digoxin, colchicine, and desipramine). ${ }^{22}$ Monoclonal antibody obtained in mice immunized with dabigatran conjugated to carrier protein were selected, the Fab fragment isolated, and a chimeric humanized fragment expressed in a mammalian cell line. Idarucizumab binds in a 1:1 ratio to the small molecule dabigatran and its active glucuronide metabolites with high affinity $(2 \mathrm{pM}$, ie, 350fold higher affinity than thrombin), thereby preventing them from thrombin inhibition. This tight binding explains the quasi-irreversible inhibition. The molecular mechanism of dabigatran capture into the cavity of the antibody binding site shares strikingly structural similarities with that of dabigatran binding to the active site of thrombin, raising the possibility that idarucizumab could mimic some of the effects of thrombin on its physiological substrates. This possibility was excluded in vitro and in vivo, and idarucizumab has no intrinsic anticoagulant or procoagulant effect and does not activate platelets. ${ }^{23}$

\section{Drug development}

Preclinical studies performed in rats and pigs pretreated with dabigatran have shown that idarucizumab not only normalizes prolonged thrombin-dependent clotting tests 
immediately after intravenous (IV) administration but also reduces excessive blood loss in a porcine trauma model. ${ }^{23}$

A pharmacokinetic study after IV bolus or 1-hour infusion of idarucizumab indicated that peak concentration $\left(C_{\max }\right)$, obtained shortly after the end of administration, and total exposure increased proportionally with dose..$^{24}$ The distribution volume at steady state was close to the blood volume $(0.06 \mathrm{~L} / \mathrm{kg})$ and idarucizumab was cleared from plasma with an initial half-life of about 45 minutes, mainly eliminated unchanged in the urine, as expected for a small protein (48 kDa). No effect on blood coagulation was observed. Preexisting antibodies cross-reacting with idarucizumab were observed in a small proportion of subjects randomized to placebo or test group, but there were no consistent antibody responses after idarucizumab administration. No hypersensitivity was observed at re-exposure 2 months later. The treatment was well tolerated and no relevant adverse events were reported. ${ }^{24}$

A Phase I trial performed on a randomized, double-blind, placebo-controlled design in healthy men, pretreated for 3 days with $220 \mathrm{mg}$ dabigatran etexilate twice daily (higher dose compared to therapeutic $150 \mathrm{mg}$ bid), showed that idarucizumab reversed dabigatran-induced anticoagulation in a dose-dependent manner and was well tolerated, with no unexpected or clinically relevant safety concerns. ${ }^{25}$ Similar results were obtained in the elderly and in subjects with renal impairment. ${ }^{26}$ Importantly, this effect was immediate and sustained for at least 24 hours for idarucizumab doses equimolar to the body load of dabigatran, indicating that dabigatran redistribution from the tissues toward blood compartment was neutralized. As idarucizumab is much larger than dabigatran, the complex was cleared by the kidney at the same rate as the free protein. Full anticoagulant effect of dabigatran was recovered 24 hours after idarucizumab administration.

Given these findings, a prospective cohort trial of idarucizumab was undertaken in patients treated with dabigatran with potential indications of urgent reversal (REVERSE-AD ClinicalTrials.gov number NCT02104947). ${ }^{27}$ In the absence of an alternative to idarucizumab with high-quality evidence, it was unethical to randomly assign patients to receive a placebo. This study is continuing, but results of an interim analysis on the first 90 patients (expected recruitment: 300 patients) have been published. ${ }^{28}$

The primary objective of this study is to evaluate the safety and ability to reverse the anticoagulant effect of dabigatran. All patients were given $5 \mathrm{~g}$ of idarucizumab as two $50 \mathrm{~mL}$ IV infusions of $2.5 \mathrm{~g}$ at 15 -minute intervals.
This dose, which was not tested in Phase II studies, was chosen to be able to reverse the total body load of dabigatran that was associated with the 99th percentile of the dabigatran levels, measured in the RE-LY trial. ${ }^{29}$ The study included two groups of patients: Group A: overt, uncontrollable, or life-threatening hemorrhage ( $\mathrm{n}=51)$; and Group B: patients who required surgery or other invasive procedures that could not be delayed for at least 8 hours and for which normal hemostasis was required $(n=39)$. Patient characteristics were representative of those included in clinical trials of dabigatran in fibrillation or venous thromboembolism and in clinical practice.

The main result is that idarucizumab rapidly and completely reversed the anticoagulant effect of dabigatran in a majority of patients with elevated clotting times (dilute TT or ecarin clotting time) at baseline. A transient increase in total dabigatran in plasma, quasi-irreversibly bound to idarucizumab and without thrombin inhibitory effect, was observed due to the redistribution of dabigatran from the extravascular spaces. However, the rare patients with a baseline concentration above the 95th percentile (>600 ng/mL), corresponding to definite overdose, ${ }^{29}$ displayed a rebound within hours following the administration of idarucizumab. ${ }^{28}$

In contrast, the efficacy on clinical outcomes is difficult, if not impossible, to assess at this stage. In Group A, this is due to the severity of the index event at enrollment and the confounding effect of intensive associated support therapy. In Group B, a majority of patients underwent surgery or invasive procedures, with reports of normal intraoperative hemostasis, but the indications of surgery were heterogeneous and with variable risks of bleeding. A major limitation of the study, besides the lack of control group, was the small patient population. It is important to note that baseline concentrations of plasma dabigatran were relatively low (median: 84 and $75 \mathrm{ng} / \mathrm{mL}$; first quartile [Q1]: 38 and $29 \mathrm{ng} / \mathrm{mL}$, in Groups A and B, respectively), corresponding to usual trough concentrations, in accordance with the time since the last intake of dabigatran being $>12$ hours in two-thirds of patients. ${ }^{29}$ A judgment about the efficacy of an antidote given when the target drug is no longer present at a concentration relevant to bleeding is hazardous. Thrombotic events were documented in five patients (5.5\%), none of them receiving antithrombotic therapy at the time of event. These events occurred 2-26 days after administration, suggesting that they were not directly linked to idarucizumab.

Therefore, the results of interim analysis confirm that idarucizumab rapidly reverses the anticoagulant effect of 
dabigatran in the usual range of plasma concentrations, and there is no signal indicating a lack of effectiveness or unexpected adverse events. These results justify the continuation of the trial to this end.

Considering that the intermediate criterion (reversion of anticoagulant effect) has a strong rationale for being associated with improved hemostasis, idarucizumab has been approved in the US and Europe under the name Praxbind ${ }^{\circledR}$ (BoehringerIngelheim) for the indications of REVERSE-AD. ${ }^{27}$ It is expected that this ultra-rapid approval would not make the enrollment in the REVERSE-AD ${ }^{28}$ clinical trial of patients with the same profile and indications more difficult. To date, idarucizumab is the only available antidote of dabigatran evaluated in a prospective trial. Of note, idarucizumab is not a reversal agent for other licensed direct thrombin inhibitors (bivalirudin and argatroban). Idarucizumab is available as a ready-to-use solution in a package containing two $50 \mathrm{~mL}$ glass vials, each containing $2.5 \mathrm{~g}$ at a concentration of $50 \mathrm{mg} / \mathrm{mL}$ (shelf life of 24 months at $2^{\circ} \mathrm{C}-8^{\circ} \mathrm{C}$ ). No reconstitution is required, which enables rapid delivery.

\section{Potential indications of idarucizumab}

The first set of indications for rapid reversal of dabigatran is bleeding from critical sites (intracranial, intraspinal, pericardial, intraocular, pulmonary, retroperitoneal, or intramuscular with compartment syndrome) or persistent major bleeding despite local hemostatic measures (gastrointestinal, gynecologic, or urologic). Life-threatening bleeding is relatively rare. With respect to nontraumatic intracranial hemorrhage, the risk is consistently reduced by about onehalf compared to VKAs, irrespective of the type of DOAC, including dabigatran, and subgroups of patients. ${ }^{30}$ The incidence of fatal hemorrhage in the RE-LY trial was 22/12,091 patients in the dabigatran arms (annual rate: 0.09\%/year vs $0.14 \%$ /year in the warfarin arm).$^{31}$

The second set of indication is emergency surgery or other invasive procedure at high risk of bleeding. ${ }^{32}$ Arbitrarily, in the REVERSE-AD trial, ${ }^{27}$ the maximal delay for patient inclusion between admission and intervention was 8 hours, and this is reproduced in the summary of product characteristics released in some countries. This should be mitigated for nonelective intervention that could be delayed for $>8$ hours in patients with reduced drug clearance (impairment of renal function, strong drug interference) or evidence of overdose at admission. A potential indication is the reversal of dabigatran in patients with acute ischemic stroke before fibrinolytic therapy. As a majority of these patients have very low anticoagulant levels at admission, in contrast with those with hemorrhagic stroke, monitoring dabigatran concentration before reversal could be particularly valuable. ${ }^{33}$ These two examples raise the question of the utility of laboratory testing in critically ill patients.

\section{Laboratory testing for idarucizumab use}

Specific assays calibrated for each of the DOACs, including dabigatran, have been developed and are now commercially available. They are equivalent to the reference method (liquid chromatography-tandem mass spectrometry) in a large range of concentrations $>50 \mathrm{ng} / \mathrm{mL}^{34}$ They can be performed in a central laboratory in a rapid turnaround time, similar to that required for the measurement of INR used for the management of patients treated by VKAs.

Rapid access to dabigatran concentration would be useful to guide reversal. When drug levels in plasma are low and unlikely to play a major role in bleeding, the administration of the antidote can be avoided. Conversely, when higher drug levels are observed, the administration of the antidote is justified. A value of $50 \mathrm{ng} / \mathrm{mL}$ can be proposed for these thresholds, without definite proof of validity. Finally, knowledge of drug concentration could help to adjust the dose of the antidote in patients with excessively high dabigatran levels.

Global coagulation tests (prothrombin time or aPTT) are not reliable for monitoring DOACs due to their low sensitivity, the lack of reagent standardization, a plateau effect of aPTT at supratherapeutic concentrations, and alternative causes of prolongation in critically ill patients. ${ }^{13,14}$ However, if the drug concentration is unknown, as far as dabigatran is concerned, normal prothrombin time and aPTT, provided these tests are performed with a sensitive reagent, indicate that the levels of dabigatran are probably low enough to perform an invasive act without additional risk due to the treatment.

The TT is exquisitely sensitive to dabigatran, and samples may be unclottable at therapeutic levels as low as $50 \mathrm{ng} / \mathrm{mL}{ }^{35}$ A normal TT indicates an absence of dabigatran and could therefore be used to exclude the drug as the cause of hemorrhage. Importantly, regular TT should not be confused with the dilute TT, which provides a measurable clotting time directly proportional to dabigatran concentrations over the therapeutic range. In REVERSE-AD, TT and dilute TT rapidly returned to the normal range after idarucizumab administration, indicating the complete absence of unbound dabigatran, but a rebound of TT and, to a lower extent, dilute TT was observed in the rare patients with overdose at baseline. ${ }^{28}$ Therefore, although being a nonspecific test, TT can be of value for monitoring incomplete reversal or ongoing bleeding after idarucizumab. 


\section{Clinical utility of idarucizumab}

The current management of dabigatran-induced bleeding, trauma, or before an emergency invasive procedure is the matter of numerous, remarkably concordant, published guidelines or expert advices. ${ }^{36-41}$ Cessation of anticoagulant treatment and administration of activated charcoal, if intake is recent, are the first measures. Supportive care is essential, with special attention to maintain renal perfusion and urine output, because dabigatran is mainly eliminated through the kidneys. Because the half-life of dabigatran is short (12-17 hours), a majority of bleeding episodes are managed by supportive methods alone. In hemodynamically stable patients, hemodialysis can be considered in case of dabigatran overdose. Mechanical methods to arrest bleeding, including compression, tamponade, surgery, and endoscopic or vascular embolization may be essential. However, if bleeding is lifethreatening or is not controlled by these measures, an additional hemostatic agent should be considered. Prothrombin complex concentrates (PCCs) or activated prothrombin complex concentrate Factor VIII inhibitor bypassing activity (FEIBA ${ }^{\circledR}$; Baxter International Inc, Deerfield, IL, USA) has been shown to improve dabigatran-induced coagulopathy in vitro and to be effective in animal models of bleeding, when given before or shortly after the induction of bleeding. ${ }^{42-45}$ Numerous case reports and short series indicate, with possible publication biases, that these agents are also effective in patients, and their use as first-line therapy in life-threatening hemorrhages and as second-line therapy in severe uncontrolled bleeding has been incorporated in all recommendations. However, these procoagulant agents have not been prospectively evaluated, and these recommendations are therefore of low level of evidence. In addition, before procoagulant agents are given to patients with a prothrombotic condition, the risk of thrombotic adverse events should be considered.

Intuitively, antidote availability is perceived as a major step toward the achievement of safer anticoagulant therapy. Antidotes for DOACs may reduce the fear of bleeding associated with oral anticoagulation and, as such, increase confidence to prescribe these drugs in relevant indications. ${ }^{46}$ However, it should be recalled that bleeding associated with VKAs, for which a specific fast-acting antidote exists (PCCs) and has been available for several years in Europe and more recently in North America, remains a major concern. In the RE-LY trial, the mortality associated with spontaneous intracerebral hemorrhage was $\sim 50 \%$, with no significant differences between the patients receiving dabigatran (no antidote available at this time) and those receiving warfarin (PCCs available). ${ }^{47}$
First, the mortality and morbidity due to a hemorrhage are, in part, attributable to underlying disorders (vascular injury, complex coagulopathy, or other issues). Second, the patient is frequently referred after bleeding has produced irreversible damage. For example, in a large randomized, placebocontrolled trial, treatment of spontaneous acute intracerebral hemorrhage with recombinant activated factor VIIa (eptacog alfa or NovoSeven ${ }^{\circledR}$, Novonordisk, Bagsvaerd, Denmark) reduced growth of the hematoma but did not improve survival or functional outcome. ${ }^{48}$ Added to the incompressible delay of patient referral, further in-hospital delay for access to treatment is frequent. In a recent multicenter observational study of medical practice, guideline-recommended administration of PCCs and vitamin $\mathrm{K}$ was associated with reduced mortality in patients on VKA therapy presenting with severe hemorrhage. ${ }^{49}$ Particularly, in patients with intracerebral hemorrhage, a threefold decrease in mortality was observed if VKA reversal was initiated at the appropriate dose within 8 hours after admission. The management was largely heterogeneous between centers and only $38 \%$ of patients received the appropriate treatment, indicating the need for improved guideline adherence. Finally, bleeding or an invasive act is associated with discontinuation of the anticoagulant for variable periods, which may be responsible for secondary thrombotic events. Therefore, a perfect antidote is only a part, maybe not the leading one, of a global strategy for patient management in these critical conditions.

\section{Conclusion}

Idarucizumab constitutes a progressive step for the safety of patients treated by dabigatran but, as it will probably not address all patients who could benefit from it, it is far from being a panacea. Emphasis should be put on good medical practice for the use of dabigatran itself: proper indications and respect of contraindications, appropriate dosing, regular control of renal function, avoiding unnecessary coadministration of interfering drugs, especially antiplatelet agents, and patient's education before prescribing the drug. The introduction of idarucizumab should be accompanied by a comprehensive effort to accelerate diagnostic procedures, improve in-hospital idarucizumab availability, as well as prepare and diffuse structured local protocols of pathways/ algorithms for the management of patients admitted in overcrowded emergency departments. ${ }^{50}$ In this manner, the promise of idarucizumab will become a reality.

\section{Disclosure}

PS has received consultancy fees, grant supports, or conference honoraria from Boehringer Ingelheim, Bayer HealthCare, 
Daiichi Sankyo, Pfizer, Bristol-Myers Squibb, LFB, Sanofi, and Diagnostica Stago. These relationships are not directly related to the content of this manuscript.

\section{References}

1. Thaler J, Pabinger I, Ay C. Anticoagulant treatment of deep vein thrombosis and pulmonary embolism: the present state of the art. Front Cardiovasc Med. 2015;2:30.

2. Verheugt FW, Granger CB. Oral anticoagulants for stroke prevention in atrial fibrillation: current status, special situations, and unmet needs. Lancet. 2015;386(9990):303-310.

3. Huhtakangas J, Tetri S, Juvela S, Saloheimo P, Bode MK, Hillbom M. Effect of increased warfarin use on warfarin-related cerebral hemorrhage: a longitudinal population-based study. Stroke. 2011;42(9): 2431-2435.

4. Wysowski DK, Nourjah P, Swartz L. Bleeding complications with warfarin use: a prevalent adverse effect resulting in regulatory action. Arch Intern Med. 2007;167(13):1414-1419.

5. Chai-Adisaksopha C, Crowther M, Isayama T, Lim W. The impact of bleeding complications in patients receiving target-specific oral anticoagulants: a systematic review and meta-analysis. Blood. 2014;124(15): 2450-2458.

6. Chai-Adisaksopha C, Hillis C, Isayama T, Lim W, Iorio A, Crowther M. Mortality outcomes in patients receiving direct oral anticoagulants: a systematic review and meta-analysis of randomized controlled trials. J Thromb Haemost. 2015;13(11):2012-2020.

7. Beyer-Westendorf J, Förster K, Pannach S, et al. Rates, management, and outcome of rivaroxaban bleeding in daily care: results from the Dresden NOAC registry. Blood. 2014;124(6):955-962.

8. Beyer-Westendorf J, Ebertz F, Förster K, et al. Effectiveness and safety of dabigatran therapy in daily-care patients with atrial fibrillation. Results from the Dresden NOAC Registry. Thromb Haemost. 2015; 113(6):1247-1257.

9. Bouillon K, Bertrand M, Maura G, Blotière PO, Ricordeau P, Zureik M. Risk of bleeding and arterial thromboembolism in patients with nonvalvular atrial fibrillation either maintained on a vitamin $\mathrm{K}$ antagonist or switched to a non-vitamin K-antagonist oral anticoagulant: a retrospective, matched-cohort study. Lancet Haematol. 2015;2(4): e150-e159.

10. Crowther M, Crowther MA. Antidotes for novel oral anticoagulants: current status and future potential. Arterioscler Thromb Vasc Biol. 2015; 35(8):1736-1745.

11. Hauel N, Nar H, Priepke H, Ries U, Stassen JM, Wienen W. Structurebased design of novel potent nonpeptide thrombin inhibitors. $J$ Med Chem. 2002;45(9):1757-1766.

12. Stangier J, Clemens A. Pharmacology, pharmacokinetics, and pharmacodynamics of dabigatran etexilate, an oral direct thrombin inhibitor. Clin Appl Thromb Hemost. 2009;15(suppl 1):9S-16S.

13. Lindahl TL, Baghaei F, Blixter IF, et al; Expert Group on Coagulation of the External Quality Assurance in Laboratory Medicine in Sweden. Effects of the oral, direct thrombin inhibitor dabigatran on five common coagulation assays. Thromb Haemost. 2011;105(2):371-378.

14. Van Ryn J, Stangier J, Haertter S, et al. Dabigatran etexilate, a novel, reversible, oral direct thrombin inhibitor: interpretation of coagulation assays and reversal of anticoagulant activity. Thromb Haemost. 2010; 103(6):1116-1127.

15. Eriksson BI, Dahl OE, Rosencher N, et al; RE-NOVATE Study Group. Dabigatran etexilate versus enoxaparin for prevention of venous thromboembolism after total hip replacement: a randomised, double-blind, non-inferiority trial. Lancet. 2007;370(9591):949-956.

16. Eriksson BI, Dahl OE, Rosencher N, et al; RE-MODEL Study Group. Oral dabigatran etexilate vs subcutaneous enoxaparin for the prevention of venous thromboembolism after total knee replacement: the RE-MODEL randomized trial. J Thromb Haemost. 2007;5:2178-2185.
17. RE-MOBILIZE Writing Committee, Ginsberg JS, Davidson BL, et al. Oral thrombin inhibitor dabigatran etexilate vs North American enoxaparin regimen for prevention of venous thromboembolism after knee arthroplasty surgery. J Arthroplasty. 2009;24(1):1-9.

18. Schulman S, Kearon C, Kakkar AK, et al; RE-COVER Study Group. Dabigatran versus warfarin in the treatment of acute venous thromboembolism. N Engl J Med. 2009;361(24):2342-2352.

19. Connolly SJ, Ezekowitz MD, Yusuf S, et al; RE-LY Steering Committee and Investigators. Dabigatran versus warfarin in patients with atrial fibrillation. N Engl J Med. 2009;361(12):1139-1151.

20. Schiele F, van Ryn J, Canada K, et al. A specific antidote for dabigatran: functional and structural characterization. Blood. 2013;121(18): 3554-3562.

21. Millar CM, Lane DA. Blocking direct inhibitor bleeding. Blood. 2013; 121(18):3543-3544.

22. Flanagan RJ, Jones AL. Fab antibody fragments: some applications in clinical toxicology. Drug Saf. 2004;27(14):1115-1133.

23. Grottke O, van Ryn J, Spronk HM, Rossaint R. Prothrombin complex concentrates and a specific antidote to dabigatran are effective ex-vivo in reversing the effects of dabigatran in an anticoagulation/liver trauma experimental model. Crit Care. 2014;18(1):R27.

24. Glund S, Moschetti V, Norris S, et al. A randomised study in healthy volunteers to investigate the safety, tolerability and pharmacokinetics of idarucizumab, a specific antidote to dabigatran. Thromb Haemost. 2015; 113:943-951.

25. Glund S, Stangier J, Schmohl M, et al. Safety, tolerability, and efficacy of idarucizumab for the reversal of the anticoagulant effect of dabigatran in healthy male volunteers: a randomised, placebo-controlled, doubleblind phase 1 trial. Lancet. 2015;386(9994):680-690.

26. Glund S, Stangier J, Schmohl M, et al. Idarucizumab, a specific antidote for dabigatran: immediate, complete and sustained reversal of dabigatran induced anticoagulation in elderly and renally impaired subjects. Blood. 2014;124(21):344. Abstract.

27. Pollack CV Jr, Reilly PA, Bernstein R, et al. Design and rationale for RE-VERSE AD: A phase 3 study of idarucizumab, a specific reversal agent for dabigatran. Thromb Haemost. 2015;114(1):198-205.

28. Pollack CV Jr, Reilly PA, Eikelboom J, et al. Idarucizumab for dabigatran reversal. $N$ Engl J Med. 2015;373(6):511-520.

29. Reilly PA, Lehr T, Haertter S, et al; RE-LY Investigators. The effect of dabigatran plasma concentrations and patient characteristics on the frequency of ischemic stroke and major bleeding in atrial fibrillation patients: the RE-LY trial (randomized evaluation of long-term anticoagulation therapy). J Am Coll Cardiol. 2014;63(4): 321-328.

30. Kundu A, Sen P, Sardar P, Chatterjee S, Kapoor A, McManus DD. Intracranial hemorrhage with target specific oral anticoagulants in patients with atrial fibrillation: an updated meta-analysis of randomized controlled trials. Int J Cardiol. 2016;15(203):1000-1002.

31. Marijon E, Le Heuzey JY, Connolly S, et al. Causes of death and influencing factors in patients with atrial fibrillation: a competing-risk analysis from the randomized evaluation of long-term anticoagulant therapy study. Circulation. 2013;128(20):2192-2201.

32. Douketis JD, Healey JS, Brueckmann M, et al. Urgent surgery or procedures in patients taking dabigatran or warfarin: analysis of perioperative outcomes from the RE-LY trial. Thromb Res. 2016;139:77-81.

33. Seiffge DJ, Hooff RJ, Nolte CH, et al; NOACISP Study Group. Recanalization therapies in acute ischemic stroke patients: impact of prior treatment with novel oral anticoagulants on bleeding complications and outcome. Circulation. 2015;132(13):1261-1269.

34. Douxfils J, Dogné JM, Mullier F, et al. Comparison of calibrated dilute thrombin time and aPTT tests with LC-MS/MS for the therapeutic monitoring of patients treated with dabigatran etexilate. Thromb Haemost. 2013;110(3):543-549.

35. Lessire S, Douxfils J, Baudar J, et al. Is thrombin time useful for the assessment of dabigatran concentrations? An in vitro and ex vivo study. Thromb Res. 2015;136(3):693-696. 
36. Kaatz S, Kouides PA, Garcia DA, et al. Guidance on the emergent reversal of oral thrombin and factor Xa inhibitors. Am J Hematol. 2012; 87(suppl 1):S141-S145.

37. Lazo-Langner A, Lang ES, Douketis J. Clinical review: clinical management of new oral anticoagulants: a structured review with emphasis on the reversal of bleeding complications. Crit Care. 2013; 17(3):230.

38. Pernod G, Albaladejo P, Godier A, et al; Working Group on Perioperative Haemostasis. Management of major bleeding complications and emergency surgery in patients on long-term treatment with direct oral anticoagulants, thrombin or factor-Xa inhibitors: proposals of the working group on perioperative haemostasis (GIHP). Arch Cardiovasc Dis. 2013;106(6-7):382-393.

39. Siegal DM, Cuker A. Reversal of novel oral anticoagulants in patients with major bleeding. J Thromb Thrombolysis. 2013;35(3):391-398.

40. Alikhan R, Rayment R, Keeling D, et al. The acute management of haemorrhage, surgery and overdose in patients receiving dabigatran. Emerg Med J. 2014;31(2):163-168.

41. Heidbuchel H, Verhamme P, Alings M, et al. Updated European heart rhythm association practical guide on the use of non-vitamin $\mathrm{K}$ antagonist anticoagulants in patients with non-valvular atrial fibrillation. Europace. 2015;17(10):1467-1507.

42. Eerenberg ES, Kamphuisen PW, Sijpkens MK, Meijers JC, Buller HR, Levi M. Reversal of rivaroxaban and dabigatran by prothrombin complex concentrate: a randomized, placebo-controlled, crossover study in healthy subjects. Circulation. 2011;124(14):1573-1579.

43. Marlu R, Hodaj E, Paris A, Albaladejo P, Cracowski JL, Pernod G. Effect of non-specific reversal agents on anticoagulant activity of dabigatran and rivaroxaban: a randomised crossover ex vivo study in healthy volunteers. Thromb Haemost. 2012;108(2):217-224.
44. Honickel M, Maron B, van Ryn J, et al. Therapy with activated prothrombin complex concentrate is effective in reducing dabigatran-associated blood loss in a porcine polytrauma model. Thromb Haemost. 2016; 115(2):271-284.

45. Zhou W, Schwarting S, Illanes S, et al. Hemostatic therapy in experimental intracerebral hemorrhage associated with the direct thrombin inhibitor dabigatran. Stroke. 2011;42(12):3594-3599.

46. Sarich TC, Seltzer JH, Berkowitz SD, et al. Novel oral anticoagulants and reversal agents: considerations for clinical development Am Heart J. 2015;169(6):751-757.

47. Hart RG, Diener HC, Yang S, et al. Intracranial hemorrhage in atrial fibrillation patients during anticoagulation with warfarin or dabigatran: the RE-LY trial. Stroke. 2012;43(6):1511-1517.

48. Mayer SA, Brun NC, Begtrup K, et al. Efficacy and safety of recombinant activated factor VII for acute intracerebral hemorrhage. $N$ Engl J Med. 2008;358:2127-2137.

49. Tazarourte K, Riou B, Tremey B, et al; EPAHK Study Group. Guidelineconcordant administration of prothrombin complex concentrate and vitamin $\mathrm{K}$ is associated with decreased mortality in patients with severe bleeding under vitamin $\mathrm{K}$ antagonist treatment (EPAHK study). Crit Care. 2014;18(2):R81.

50. Kulstad EB, Sikka R, Sweis RT, Kelley KM, Rzechula KH. ED overcrowding is associated with an increased frequency of medication errors. Am J Emerg Med. 2010;28(3):304-309.
Drug Design, Development and Therapy

\section{Publish your work in this journal}

Drug Design, Development and Therapy is an international, peerreviewed open-access journal that spans the spectrum of drug design and development through to clinical applications. Clinical outcomes, patient safety, and programs for the development and effective, safe, and sustained use of medicines are a feature of the journal, which

\section{Dovepress}

has also been accepted for indexing on PubMed Central. The manuscript management system is completely online and includes a very quick and fair peer-review system, which is all easy to use. Visit http://www.dovepress.com/testimonials.php to read real quotes from published authors.

Submit your manuscript here: http://www.dovepress.com/drug-design-development-and-therapy-journal 